\title{
Performance Analysis of SDL Systems
}

\author{
Mihal Brumbulli and Emmanuel Gaudin
}

\begin{abstract}
The increasing complexity of software systems is constantly fueling the interest in pragmatic analysis methods. These are by no means scarce, but their applicability requires additional expertise that often has a weak relation with the development process or the domain the system is intended for. The model-driven paradigm addresses this issue at a certain extent by raising the level of abstraction closer to the domain and facilitating development and analysis by means of automation. It tries to shift the inherent complexity from the model towards the automation process. Although this has proven to be quite effective in handling functional aspects, the same cannot be stated with confidence regarding non-functional aspects like performance. In this paper we present a model-driven approach for performance analysis based on standardized languages. The functional aspects of the system are captured using SDL and enriched with performance annotations. Available resources are assigned to system components via deployment diagrams, and real test cases described in TTCN-3 drive model execution. Different scenarios can be executed automatically, and the graphical presentation of results can aid the user to decide on the best allocation of resources in terms of execution time and payload.
\end{abstract}

M. Brumbulli (汭 · E. Gaudin (汭

PragmaDev, 18 Rue Des Tournelles, 75011 Paris, France

e-mail: mihal.brumbulli@pragmadev.com

E. Gaudin

e-mail: emmanuel.gaudin@pragmadev.com

G. Fanmuy et al. (eds.), Complex Systems Design \& Management,

DOI 10.1007/978-3-319-49103-5_19 